\title{
Band structure and optical properties of germanium sheet polymers
}

\author{
Zoltán Hajnal, ${ }^{1,2}$ Günther Vogg, ${ }^{3}$ Lex J.-P. Meyer, ${ }^{3}$ Bernadett Szúcs, ${ }^{4}$ Martin S. Brandt, ${ }^{3}$ and Thomas Frauenheim ${ }^{1}$ \\ ${ }^{1}$ Department of Theoretical Physics, University of Paderborn, Warburger Strasse 100, D-33095 Paderborn, Germany \\ ${ }^{2}$ MTA Research Institute for Technical Physics and Materials Science of the Hungarian Academy of Sciences, \\ P.O. Box 49, H-1525 Budapest, Hungary \\ ${ }^{3}$ Walter Schottky Institut, Technische Universität München, Am Coulombwall, D-85748 Garching, Germany \\ ${ }^{4}$ Department of Atomic Physics, Budapest University of Technology and Economics, Budafoki út 8, H-1111 Budapest, Hungary
}

(Received 23 April 2001; published 25 June 2001)

\begin{abstract}
The band structure of H-terminated Ge sheet polymers is calculated using density-functional theory in the local density approximation and compared to the optical properties of epitaxial polygermyne layers as determined from reflection, photoluminescence, and photoluminescence excitation measurements. A direct band gap of $1.7 \mathrm{eV}$ is predicted and a near resonant excitation of the photoluminescence is observed experimentally close to this energy.
\end{abstract}

DOI: 10.1103/PhysRevB.64.033311

PACS number(s): 78.20.Bh, 73.20.At, 78.55.-m, 78.66. $-\mathrm{w}$

The dimensionality of physical systems can have a pronounced influence on their properties. A prominent example from solid state physics is carbon, where the electronic properties of diamond, graphite, nanotubes, and fullerenes, which extend in three, two, one, and zero dimensions, respectively, differ significantly. The latter three modifications are based on bonding between $s p^{2}$-hybridized orbitals that can be formed only in exceptional cases by the other group-IV atoms. However, modifications with planar or linear structures formed by $\mathrm{Si}$ or Ge can be obtained by terminating $s p^{3}$ orbitals not needed for the construction of the network with $\mathrm{H}$ atoms. In the case of $\mathrm{Si}$, sheet polymers such as siloxene ${ }^{1}$ and chain polymers such as polysilane ${ }^{2}$ are well known. The difference in the properties of group-IV networks with different dimensionalities is particularly evident when looking at the electronic band structure: ${ }^{3}$ While crystalline silicon has an indirect band gap (with the minimum of the conduction band near the $X$ point of the Brillouin zone) and only shows an extremely weak photoluminescence, ${ }^{4}$ silicon sheet and chain polymers have a direct band gap resulting in a strong, visible, ${ }^{5-9}$ and near-ultraviolet photoluminescence, ${ }^{2}$ respectively. A similar dependence of the band structure on the dimensionality of the network is found for germanium: Crystalline Ge again has an indirect band gap (with the minimum of the conduction band at the $L$ point of the Brillouin zone) and a very weak photoluminescence. ${ }^{4}$ Chain polymers of $\mathrm{Ge}$ such as polygermane also have a strong ultraviolet luminescence, ${ }^{10,11}$ which is shifted to lower energies with respect to the corresponding polysilanes. Theoretical investigations have shown that these linear Ge polymers also have a direct band gap. ${ }^{12}$ However, for a full investigation of the influence of the dimensionality of the Ge network on the electronic properties, both experimental and theoretical data on planar germanium sheet polymers have been missing. Here, we show using density-functional calculations as well as experimental investigations of the optical properties that the Ge sheet polymers also have a band structure with a direct gap which is redshifted to about $1.7 \mathrm{eV}$ with respect to the band gap of the corresponding Si sheet polymers.

Attempts to form Si or Ge sheet polymers by polymerization have until now only led to amorphous network structures. ${ }^{13,14}$ Ordered stacks of extended layers can, however, be obtained by a topochemical transformation of the Zintl phases calcium-disilicide $\left(\mathrm{CaSi}_{2}\right)$ (Ref. 15) or calciumdigermanide $\left(\mathrm{CaGe}_{2}\right)$ (Ref. 16). These Zintl phases consist of alternating layers of $\mathrm{Ca}$ and $\mathrm{Si}$ or $\mathrm{Ge}$, respectively, where the group-IV layers are isomorphic to the $\{111\}$ double layers in the diamond crystal structure of the elemental semiconductors. ${ }^{17,18}$ Using aquaeus hydrochloric acid, Ca can be removed from the Zintl phases, and the fourth valence of each group-IV atom is terminated with $\mathrm{H}$ or $\mathrm{OH}$ groups. As a result, sheet polymers such as polysilyne $(\mathrm{SiH})_{n},{ }^{19}$ siloxene $(\mathrm{SiHSiOH})_{n}$, and polygermyne $(\mathrm{GeH})_{n}$ are obtained with the "back sheet" consisting of the $\{111\}$ double layers discussed above. The stacking sequence of the Zintl phases is preserved in the polymer crystals, so that predominantly a trigonal-rhombohedral modification with a sixfold stacking sequence (tr6) of the polymers is formed. ${ }^{1,16}$

For the determination of the optical properties of the Ge and $\mathrm{Si}$ sheet polymers studied here, epitaxial polymer films on crystalline $\mathrm{Si}$ or $\mathrm{Ge}$ were investigated. $\mathrm{CaGe}_{2}$ and $\mathrm{CaSi}_{2}$ were first grown via reactive deposition epitaxy on (111)oriented germanium and silicon substrates, respectively, ${ }^{20,21}$ and topochemically transformed in concentrated $\mathrm{HCl}$ at $-30{ }^{\circ} \mathrm{C} .{ }^{16,15}$ The polymer layers obtained are epitaxial on the substrates with the planes oriented parallel to the (111) surface of the substrates. ${ }^{16,21}$ While pure polygermyne sheet polymers exclusively with $\mathrm{H}$ bonded to the back sheet are formed this way, the corresponding $\mathrm{Si}$ sheet polymers still contain a significant amount of $\mathrm{OH}$ groups also at this low transformation temperature, in contrast to earlier reports. ${ }^{22}$ We therefore refer to them as siloxene. The photoluminescence (PL) experiments were performed at $77 \mathrm{~K}$ using the $457 \mathrm{~nm}$ and $363 \mathrm{~nm}$ lines of an $\mathrm{Ar}^{+}$laser for the excitation of polygermyne and siloxene, respectively. For the determination of the efficiency of the photoluminescence excitation (PLE), the PL was excited with light from a tungsten lamp filtered by a monochromator and the luminescence intensity at a fixed detection wavelength was measured as a function of the excitation wavelength. The diffuse reflectivity $R$ was measured with an ultraviolet-visible range spectrometer using an Ulbricht sphere for integration and polytetrafluoroethene (PTFE) as a standard. All data were corrected for spectrometer response. 
For the calculation of the electronic band structure of the sheet polymers, we use the local density approximation (LDA) to the density functional theory ${ }^{23}$ (DFT), which has been shown to provide reliable predictions of the energetics and electronic properties over a wide range of materials both in the molecular as well as in the solid state. From many existing implementations of the DFT, we have chosen the FHI98MD code ${ }^{24}$ for its capability of handling periodic models, using localized [linear combination of atomic orbitals (LCAO)] as well as plane-wave (PW) basis sets for the expansion of the valance electron density. We have employed a mixed LCAO/PW basis set for the initial density calculation, and used a 48 Ry kinetic energy cutoff of the PW's in the geometry optimization through total energy minimization and the consecutive band structure calculations. The ion cores were represented by the norm-conserving pseudopotentials (PP) constructed according to Hamann's generalized method. ${ }^{25}$

First, to test the potentials used in our calculations, we have performed some band structure and elasticity calculations on bulk crystalline $\mathrm{Ge}$ and $\mathrm{Si}$. The calculated Ge band structure shows a disappearing gap at the center of the Brillouin zone (BZ). This behavior is known for the LDA, ${ }^{26,27}$ and can only be corrected by costly quasiparticle $(G W)$ calculations. The usual way to circumvent the underestimation of the band gap is to lift the conduction band states' energy by a constant amount (uniformly across the BZ) so that the experimentally observed gap value is obtained (scissors operator). Therefore, in Ge-based systems we use a scissors operator of $0.7 \mathrm{eV}$, and $0.6 \mathrm{eV}$ in Si-based systems, as our LDA calculations predict an indirect gap of $\sim 0.5 \mathrm{eV}$ for crystalline $\mathrm{Si}$. The calculated equilibrium $\mathrm{Ge}-\mathrm{Ge}$ bond length is $2.42 \AA$, in good agreement with the experimental value of $2.45 \AA{ }^{28}$ Also, the calculated bulk moduli of $68 \mathrm{GPa}$ for Ge and $88 \mathrm{GPa}$ for $\mathrm{Si}$ agree well with the experimental values of 77 and $99 \mathrm{GPa},{ }^{29}$ respectively. These results indicate that the method and potentials can be used for predictive calculations on less studied group-IV structures as well.

Since only H-terminated germanium sheet polymers have been prepared so far, we restrict ourselves to the calculation of the electronic band structure of pure polygermyne. To determine the effect of different group-IV atoms in the back sheet, we will compare these results to the band structure of the corresponding $\mathrm{H}$-terminated polysilyne. The sheet polymer layers have been modeled by the periodic repetition of the hexagonal unit cell identified by x-ray diffraction containing two $\mathrm{Ge}$ and two $\mathrm{H}$ atoms as shown in Fig. 1. 16,30 However, in contrast to the sixfold stacking (tr6 modification) observed in x-ray diffraction, a simple one-on-one stacking of the unit cells is used for the calculation, corresponding to an $\mathrm{h} 1$ modification. Previous theoretical work on polymers has indeed shown that the band structure is predominantly determined by the atomic configuration within the layers and rather insensitive to interlayer interactions. ${ }^{7,8}$ The atomic coordinates and the lateral in-plane lattice constant $a$ have been optimized by minimizing the computed total energy. The atomic distances and lattice constants obtained from this energy minimization are compared to the experimentally observed values in Table I. For the band

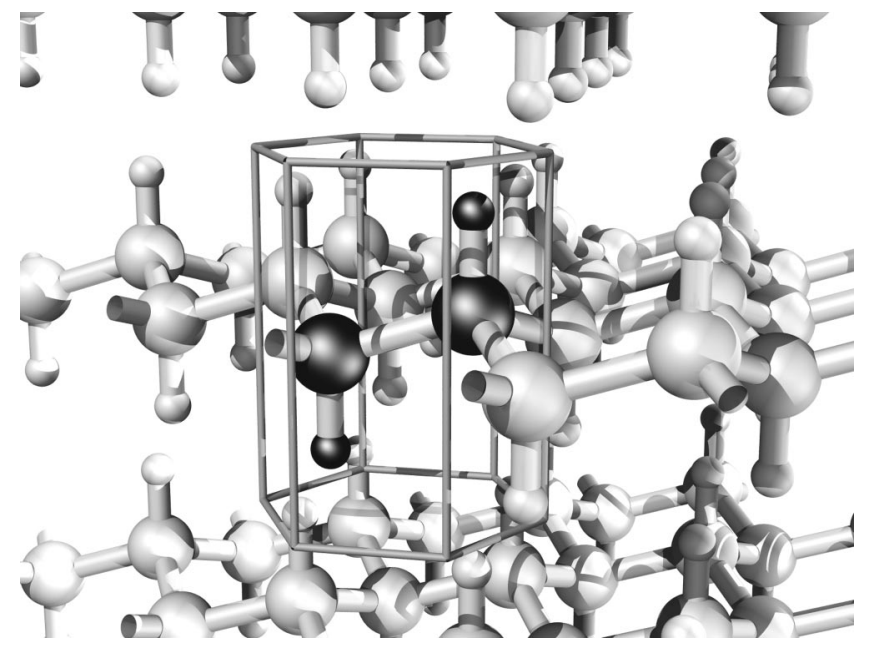

FIG. 1. Model structure of the H-terminated $\mathrm{Si}$ and $\mathrm{Ge}$ sheet polymers. The stacking shown corresponds to a simple one-on-one stacking (h1 modification). The unit cell (atoms shaded dark) is marked by the hexagonal prism.

structure calculation, we used the interlayer distance as determined by x-ray diffraction. ${ }^{16,30}$

The calculated band structures for polygermyne and polysilyne are compared in Figs. 2 and 3 using the conventional notation for the symmetry points in a hexagonal Brillouin zone. The known LDA-DFT result for polysilyne (dashed curves), namely a band structure with an indirect gap of 2.7 $\mathrm{eV}$ between the valence band maximum at $\Gamma$ and a conduction band minimum close to $M$ is well reproduced. The smallest direct band gap $(3.0 \mathrm{eV})$ is found at the $\Gamma$ point. These values are in good agreement with the results of Van de Walle and Northrup, ${ }^{8}$ who have determined 2.75 and 2.95 $\mathrm{eV}$, respectively, by a $G W$ calculation. This supports our use of the scissors operator also for the calculation of polymer band structures, showing that the self-energy correction can be well approximated by a uniform shift of the conduction band levels based on the LDA error for the band gap of the elemental semiconductors.

Now, looking at the energy bands of polygermyne (solid curves), one immediately notices a qualitative similarity of the valence bands to those of polysilyne. The most relevant

TABLE I. Structural parameters of the Si- and Ge-based sheet polymers. $a$ is the in-plane lattice constant, $c$ the distance between neighboring polymer sheets (corresponding to $1 / 6$ of the lattice constant in the $c$ direction for the tr6 modifications). $X$ stands for either $\mathrm{Si}$ or Ge in the bond length labels $X-X$ and $X-\mathrm{H}$.

\begin{tabular}{|c|c|c|}
\hline & Polysilyne & Polygermyne \\
\hline$a$ (theor.) & $3.82 \AA$ & $3.94 \AA$ \\
\hline$a(\text { expt. })^{\mathrm{a}}$ & $3.84 \AA$ & $3.98 \AA$ \\
\hline$c(\text { expt. })^{\mathrm{a}}$ & $5.57 \AA$ & $5.65 \AA$ \\
\hline$X-X$ (theor.) & $2.32 \AA$ & $2.39 \AA$ \\
\hline$X-\mathrm{H}$ (theor.) & $1.52 \AA$ & $1.54 \AA$ \\
\hline
\end{tabular}

${ }^{\mathrm{a}}$ The experimental data for polysilyne and polygermyne are from Refs. 30 and 16, respectively. 


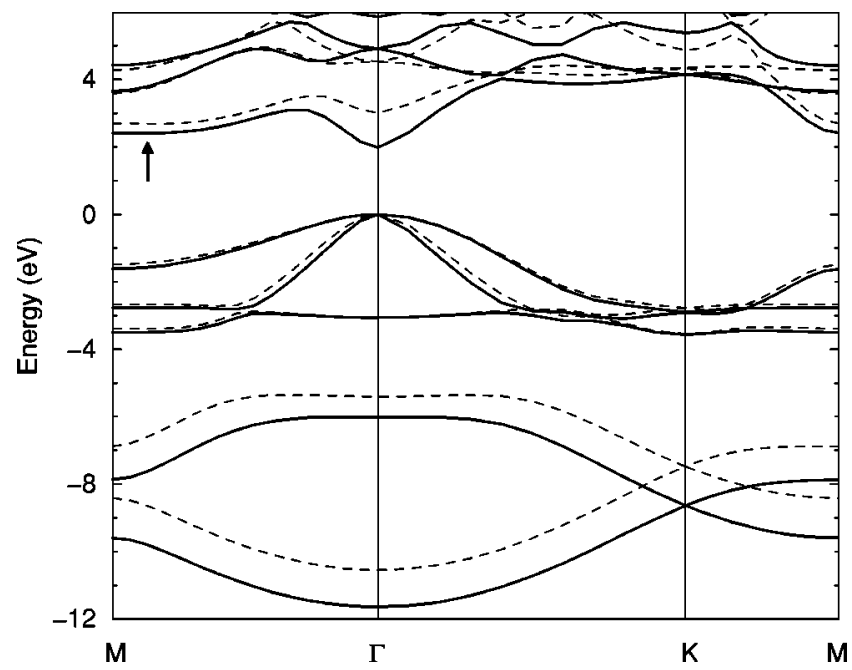

FIG. 2. Band structure of H-terminated Ge (solid lines) and Si (dashed lines) sheet polymers in the basal plane. The arrow marks the position of the lowest energy point of the conduction band edge in polysilyne.

difference in the band structure can be seen at the zone center $(\Gamma)$. Here, the first conduction band has a deep minimum in the $M-\Gamma-K$ plane with a calculated energy of the direct transition of $2.0 \mathrm{eV}$. However, this is not the global minimum of the conduction band. Figure 3 shows that the first conduction band of polygermyne rather has a minimum at the $A$ point (zone boundary in the direction perpendicular to the plane of the two-dimensional polymer). Since the valence band edge shows practically no dispersion in that direction, we obtain a predicted direct gap at $A$ of $1.7 \mathrm{eV}$. However, for the calculation we have assumed a simple one-on-one stacking of the polymer layers, while the topochemically formed polygermyne films show a sixfold stacking sequence. The band structures for these modifications are obtained by a folding of the band structure shown in Fig. 3 in the $\Gamma-A$ direction. Thus we conclude that the computed transition at

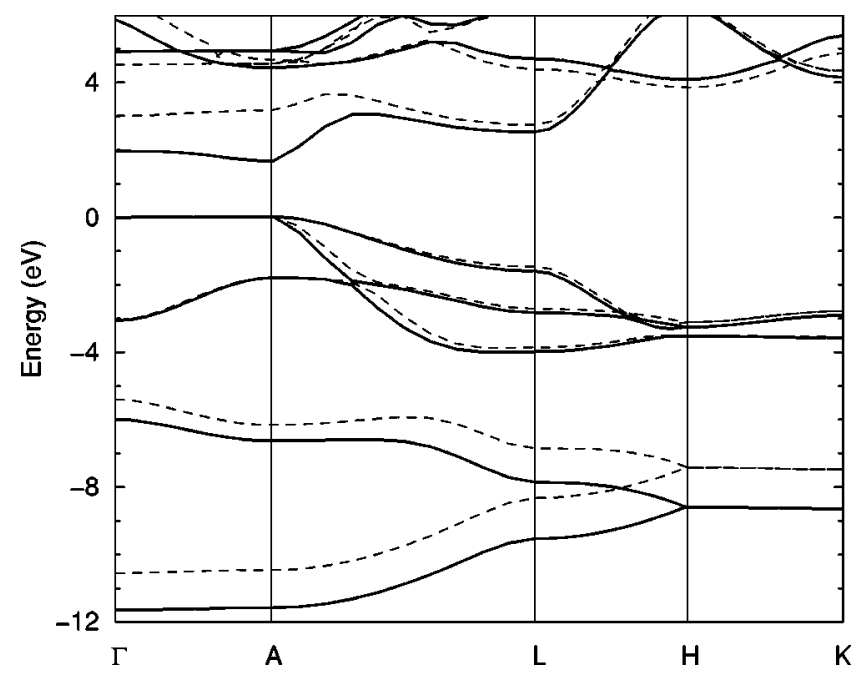

FIG. 3. Band structure of H-terminated Ge (solid lines) and $\mathrm{Si}$ (dashed lines) sheet polymers out of the basal plane. A direct band gap of $1.7 \mathrm{eV}$ is found for polygermyne at the $A$ point.

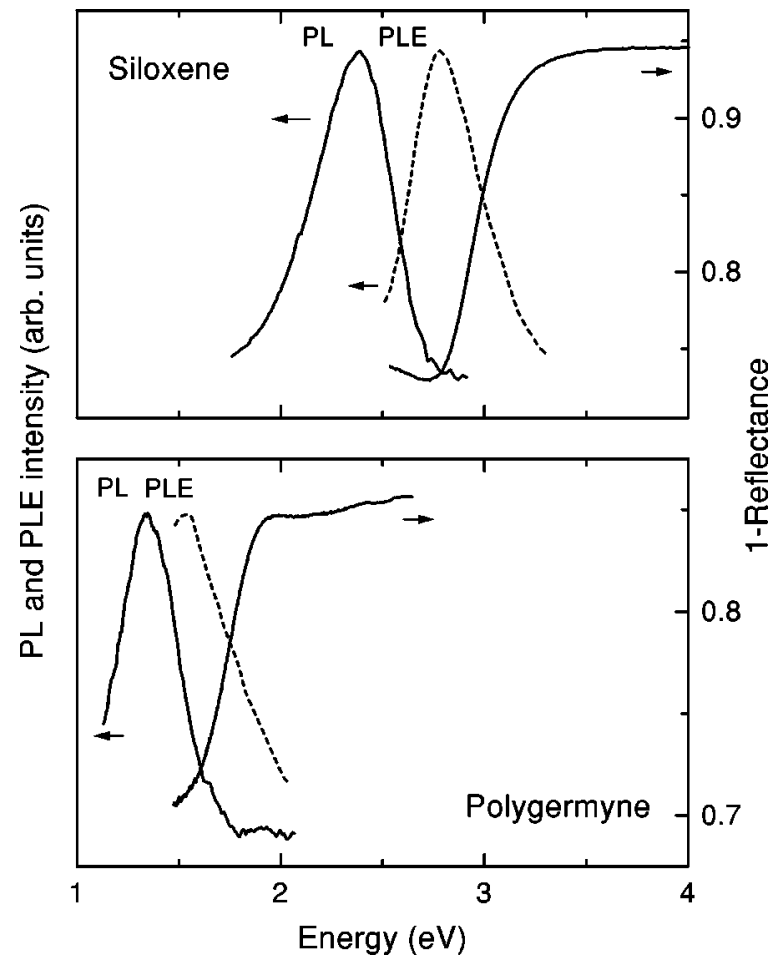

FIG. 4. Photoluminescence, photoluminescence excitation (dashed line), and reflectance spectra of siloxene (upper part) and polygermyne (lower part).

$A$ actually takes place at the $\Gamma$ point in the real tr6 phase. The same holds for the minority $\mathrm{h} 2$ modification of polygermyne. ${ }^{16}$

The experimentally observed optical properties of the sheet polymers investigated are compiled in Fig. 4. Polygermyne has a strong photoluminescence (external luminescence efficiency $\approx 1 \%$ ) centered around $1.35 \mathrm{eV}$, with the photoluminescence band extending up to an energy of about $1.7 \mathrm{eV}$. At this energy, the reflectance $R$ shows a sudden change. Since the crystalline substrate effectively works as a mirror, $1-R$ can be used to estimate the absorption of the epilayer. Comparative direct measurements of the absorption by photothermal deflection spectroscopy (PDS) show that polygermyne has an absorption coefficient of $\approx 10^{4} \mathrm{~cm}^{-1}$ at $1.7 \mathrm{eV}$. The dashed line shows the excitation efficiency (PLE) of the luminescence in the PL maximum. The small Stokes shift between the PL and PLE maxima of $0.2 \mathrm{eV}$ indicates a nearly resonant excitation of the PL of polygermyne. Siloxene has a highly similar behavior: The photoluminescence (external efficiency $>1 \%$ ) with a maximum intensity at $2.4 \mathrm{eV}$ extends to $2.7 \mathrm{eV}$, where $1-R$ and the absorption also show a steplike increase [absorption coefficient of $2 \times 10^{4} \mathrm{~cm}^{-1}$ at $2.8 \mathrm{eV}$ from PDS (Ref. 31)]. The Stokes shift of $0.35 \mathrm{eV}$ found for the epilayers is similar to the values reported for bulk material. ${ }^{6,32}$ While pure polysilyne is predicted to have an indirect band gap, when about $50 \%$ of the $\mathrm{H}$ atoms are substituted by $\mathrm{OH}$ groups in siloxene, the conduction band at $\Gamma$ is lowered significantly and a direct gap is formed. ${ }^{8}$ In agreement with these theoretical predictions, the experimental results summarized as well as time-resolved measurements of the polarization memory of 
the luminescence ${ }^{6}$ show that siloxene has a band structure with a direct gap. The intense photoluminescence, absorption edge, and small Stokes shift of polygermyne together with the strong similarity to the behavior of siloxene therefore are clear experimental evidence that polygermyne also has a direct band gap, however, with an energy of about $1.7 \mathrm{eV}$. The significantly smaller Stokes shift of the Ge sheet polymers could be due to the missing chemical disorder of the $\mathrm{OH}$ groups, which are randomly bonded to the back sheet in siloxene, but are absent in polygermyne.

In conclusion, we have calculated the electronic band structure of polygermyne using LDA-DFT. In the simple, hypothetical h1 modification, a direct band gap of $1.7 \mathrm{eV}$ is found at the $A$ point, which is folded to the central $\Gamma$ point in the tr6 and h2 modifications. Reflection and absorption mea- surements show that polygermyne indeed has a band gap at this energy and the near-resonant excitation of the photoluminescence supports the assignment to a direct gap. This detailed comparison of the optical properties with the calculated band structure shows that Ge sheet polymers could also be used as very efficient light emitters in active optoelectronic devices. The complete miscibility of $\mathrm{Si}$ and $\mathrm{Ge}$ in crystalline and amorphous materials suggests that similar mixed Si-Ge sheet polymers would also exhibit band structures with a direct gap.

The authors gratefully acknowledge financial support by the Deutsche Forschungsgemeinschaft through Schwerpunkt "Silicium-Chemie'” (Br 1585/2-3 and Fr889/9-3).
${ }^{1}$ A. Weiss, G. Beil, and H. Meyer, Z. Naturforsch. B 35b, 25 (1979).

${ }^{2}$ R. D. Miller and J. Michl, Chem. Rev. 89, 1359 (1989).

${ }^{3}$ N. Matsumoto, K. Takeda, H. Teramae, and M. Fujino, Adv. Chem. Ser. 224, 515 (1990).

${ }^{4}$ J. Weber and M. I. Alonso, Phys. Rev. B 40, 5683 (1989).

${ }^{5}$ H. Kautsky and G. Herzberg, Z. Anorg. Chem. 139, 135 (1924).

${ }^{6}$ I. Hirabayashi, K. Morigaki, and S. Yamanaka, J. Non-Cryst. Solids 59\&60, 645 (1983).

${ }^{7}$ K. Takeda and K. Shiraishi, Phys. Rev. B 39, 11028 (1989).

${ }^{8}$ C. G. Van de Walle and J. E. Northrup, Phys. Rev. Lett. 70, 1116 (1993).

${ }^{9}$ P. Deák, M. Rosenbauer, M. Stutzmann, J. Weber, and M. S. Brandt, Phys. Rev. Lett. 69, 2531 (1992).

${ }^{10}$ P. Trefonas and R. West, J. Polym. Sci., Polym. Chem. Ed. 23, 2099 (1985).

${ }^{11}$ R. D. Miller and R. Sooriyakumaran, J. Polym. Sci., Part A: Polym. Chem. 25, 111 (1987).

${ }^{12}$ K. Takeda, J. Phys. Soc. Jpn. 63, Suppl. B 1 (1994).

${ }^{13}$ A. Kleewein and H. Stüger, Chem. Monthly 130, 69 (1999).

${ }^{14}$ H. Kishida, H. Tachibana, M. Matsumoto, and Y. Tokura, Appl. Phys. Lett. 65, 1358 (1994).

${ }^{15}$ F. Wöhler, Liebigs Ann. Chem. 127, 257 (1863).

${ }^{16}$ G. Vogg, M. S. Brandt, and M. Stutzmann, Adv. Mater. 12, 1278 (2000).

${ }^{17}$ Strukturberichte 1, 175 (1913-28).
${ }^{18}$ H. J. Wallbaum, Naturwissenschaften 32, 76 (1944).

${ }^{19}$ G. Schott, Z. Chem. (Leipzig) 3, 41 (1963).

${ }^{20}$ G. Vogg, M. S. Brandt, M. Stutzmann, I. Genchev, A. Bergmaier, L. Görgens, and G. Dollinger, J. Cryst. Growth 212, 148 (2000).

${ }^{21}$ G. Vogg, M. S. Brandt, M. Stutzmann, and M. Albrecht, J. Cryst. Growth 203, 570 (1999).

${ }^{22}$ S. Yamanaka, H. Matsu-ura, and M. Ishikawa, Mater. Res. Bull. 31, 307 (1996).

${ }^{23}$ P. Hohenberg and W. Kohn, Phys. Rev. 136, B864 (1964).

${ }^{24}$ M. Bockstedte, A. Kley, J. Neugebauer, and M. Scheffler, Comput. Phys. Commun. 107, 187 (1997).

${ }^{25}$ D. R. Hamann, Phys. Rev. B 40, 2980 (1989).

${ }^{26}$ M. Rohlfing, P. Krüger, and J. Pollmann, Phys. Rev. B 48, 17791 (1993).

${ }^{27}$ I. N. Remediakis and E. Kaxiras, Phys. Rev. B 59, 5536 (1999).

${ }^{28}$ Structure Data of Elements and Intermetallic Phases, LandoltBörnstein Volume III/6, 2.1 (Springer-Verlag, Berlin, 1971).

${ }^{29}$ Elastic, Piezoelectric, Piezooptic and Electrooptic Constants of Crystals, Landolt-Börnstein Volume III/1, 1.2 (Springer-Verlag, Berlin, 1966).

${ }^{30}$ U. Dettlaff-Weglikowska, W. Hönle, A. Molassioti-Dohms, S. Finkbeiner, and J. Weber, Phys. Rev. B 56, 13132 (1997).

${ }^{31}$ M. S. Brandt, T. Puchert, and M. Stutzmann, Solid State Commun. 102, 365 (1997).

${ }^{32}$ M. Stutzmann, M. S. Brandt, M. Rosenbauer, J. Weber, and H. D. Fuchs, Phys. Rev. B 47, 4806 (1993). 